\title{
Humanistic Mathematics Network Journal
}

Issue 5

Article 5

5-1-1990

\section{Heuristic Thinking and Mathematics}

J. F. Lucas

University of Wisconsin, Oshkosh

Follow this and additional works at: http://scholarship.claremont.edu/hmnj

Part of the Curriculum and Instruction Commons, Mathematics Commons, and the Science and Mathematics Education Commons

\section{Recommended Citation}

Lucas, J. F. (1990) "Heuristic Thinking and Mathematics," Humanistic Mathematics Network Journal: Iss. 5, Article 5. Available at: http://scholarship.claremont.edu/hmnj/vol1/iss5/5

This Article is brought to you for free and open access by the Journals at Claremont at Scholarship @ Claremont. It has been accepted for inclusion in Humanistic Mathematics Network Journal by an authorized administrator of Scholarship @ Claremont. For more information, please contact scholarship@cuc.claremont.edu. 


\title{
HEURISTIC THINKING AND MATHEMATICS
}

\author{
MAA Contributed Paper \\ Humanistic Mathematics \\ 72nd Annual Meeting \\ Phoenix, Arizona \\ January 11-12, 1989 \\ J. F. Lucas \\ Mathematics Department \\ University of Wisconsin-Oshkosh
}

\section{Thesis}

Mathematics is created by human beings; it is a monumental artifact of human reasoning. Those who would study this field seriously need to know not only mathematical results, but also the reasoning processes by which these results are achieved. Human reasoning processes are the cement that holds key ideas together as well as the conduit through which information is generated. Among these reasoning processes are heuristics, or methods for inventing problem solutions. One who understands and uses heuristics sees better the cohesiveness of the subject and is more capable of discovering new information about it. For example, we can chunk whole courses down into a handful of key related ideas. Calculus is function, limit, derivative, integral and fundamental theorem. Linear algebra is vector space, basis, matrix and linear transformation. We can also take a single idea such as rate of change in calculus or vector in linear algebra and produce many different instances that embody the same fundamental idea. So, in the process of doing mathematics, we move information around; we regroup old information, we generate new information, and we manage it and mold it like a sculptor to suit our needs and to satisfy our aesthetic sense. In our models, we emphasize form instead of content, yet we expect our students to intuitively understand what that means. This uncanny ability humans possess to manage information should be a focal point of mathematical instruction. It should not be regarded as incidental subject matter which might be picked up by perceptive students. Some say the only way to become a good problem solver is to solve lots of problems. Hopefully, they mean that solving lots of problems is a necessary but not sufficient condition for becoming a good problem solver. Unfortunately, too many mathematics professors hold the opinion that their students will deduce or induce the important strategies of problem solving just by observation - the "immersion/osmosis" approach. This may be humanizing mathematics by requiring considerable human effort, but it is unrealistic in that it fails to stress those cognitive behaviors that uniquely characterize the human aspect of the development of mathematics.

Before our students can achieve real learning of mathematics, they need to develop a proper perspective on the nature of the subject, itself. There is a folklore about mathematics, and in this folklore are certain myths that bias their view on how to perceive and learn the subject.

\section{Myths}

Here are five common myths that tend to block mathematical learning:

\section{Mrth\#1}

Mathematics is a collection of isolated facts and tricky techniques that must be memorized.

This perception of mathematics overwhelms people. It is fostered by texts and teachers that present the subject in a fragmented way - by classifying problems and categorizing techniques for handling specitic kinds of problems. A better perception is to visualize mathematics as a network of related ideas with means of moving to and from these ideas. It helps to understand that no problem exists in a vacuum there are other problems related in some way to any given problem. Some memorization is undoubtedly necessary, but searching for ways to minimize memorization has the effect of maximizing the understanding of relationships between ideas. 


\section{Myth \#2}

\section{Mathematical tnith is absolute.}

Those who would believe this have great difficulty being flexible. They also ascribe to mathematics a superhuman quality. Belief in a principle is good, but unwillingness to change our beliefs in the face of compelling evidence is foolish. We must always strive to answer the question "Why?" and dare to ask the question "What if...?"

\section{Myth\#3}

\section{Mathematics is an exact science.}

This is a popular layman's belief. Mathematics deals with numbers; numbers are exact; therefore, mathematics is exact. 'You either get the right answer or you don't." But we who work with mathematics know differently. Even in the theoretical foundations of mathematics, there are unresolved questions about the axioms. And when the axioms change, the mathematics changes. Moreover, whenever mathematics is used to model reality, approximation and estimation become the norm rather than the exception.

\section{Myth\#4}

Mathematics deals primarily with symbolic representation and manipulation. so ordinary writine and speaking skills are not necessary for communicating mathematics.

Anyone who has graded written problem solutions or proofs or who has tried to evaluate oral presentations in mathematical topics has felt firsthand the effect of this attitude. While mathematics, like music or art, can be correctly regarded as a universal language, its clear communication in any culture depends on carefully constructed written and spoken language. As more mathematics develops, technical writing skill and precise verbal communication become increasingly essential to the user and doer of mathematics. One of the largest problems facing industry today is the inability of technical people to communicate.

\section{Myth \#5}

Mathematics is something one does alone.

This is only a partial truth. True, some mathematics is perhaps most efficiently handled by individuals (e.g. intensely focused problem solving). But more often than not, complex problems requiring mathematical solutions are being resolved by teams and think tanks. In many instances, sharing ideas and teamwork enhances individual creativity rather than stifling it. Often it is simply too inefficient to try to produce an effective solution by relying only on individual inputs.

Some or all of these myths characterize the beliefs of our students about the nature of mathematics. As a condition of mathematical learning, we need to dispel such myths. Our students need to view mathematics as a cohesive body of knowledge. They need to challenge their own beliefs, to place a value on approximation, to communicate clearly and precisely, to learn to work together, and to incorporate mathematical heuristics into their own thinking.

\section{Heuristics}

George Polya defined "heuristic" as a rule of thumb or method by which a person invents a solution to a problem. The method of solution itself highlights the principal difference between an exercise and a problem. An exercise requires little, if any, creative thinking, except to classify it, identify a suitable algorithm, and execute the algorithm. A problem, on the other hand, requires that a method be invented, and its solution depends heavily on certain thinking strategies of the solver. There is a parallel distinction between algorithm and heuristic. An algorithm is a stepwise procedure that is class or problem-specific and which guarantees success if the steps are taken carefully. Heuristics, by contrast, transcend classes of problems, but they are tentative processes with no guarantee of success. For example, there is no guarantee that drawing a diagram will really help us solve a given problem. But there is some strength to the belief that application of heuristics over the long run increases our probability of success. Some heuristics identified by Polya which seem to characterize the mathematical thinking of experts are the following: devising a model, using analogies to discover related problems, searching for patterns, working backwards, applying successive approximation, reducing a problem to a simpler case, extending a problem by generalization, exploiting symmetry, checking, solving a problem in several different ways, and studying a correctly solved problem. Following Polya's method of teaching by inquiry, heuristics are best illustrated by asking lead- 
ing questions that guide the problem solver to think in a certain way. Here are some examples:

(1) We know from geometry the relationship between a tangent and radial line intersecting on a circle is that of perpendicularity. Are there any similarities with respect to ellipses and hyperbolas?

(2) Solving a quadratic equation with real coefficients is easy. What if we change the coefficients to complex numbers?

(3) We are to prove: Given an $e>0$, we can find $\partial>0$, such that $|f(x)-L|<e$ whenever $0<|x-a|<\partial$. Where do we start? How do we chain our reasoning?

(4) Our problem is to devise a formula for counting the number of elements in $\mathrm{k}$ sets. What makes this problem difficult? Can we make it into an easier problem?

(5) Suppose you know the number of space compartments formed by k planes in general position. What is the effect of adding one more plane? How many new compartments are formed? Is there a related situation in lower dimension that may help?

(6) We want a fresh perspective on voting patterns in an election. Suppose we think of political issues as dimensions and voters as points in a geometric "issue space." Does this help us to understand or predict voting patterns?

(7) By suitable integration, we find that the area of an ellipse with semidiameters $\mathbf{a}$ and $\mathbf{k}$, is $\pi \mathrm{ab}$. Is this result reasonable? Can we check this answer in different ways?

(8) We are to find an integer a such that the polynomial $x^{2}-x+a$ divides the polynomial $x^{13}+$ $x+90$. Obviously, one method is to try various values for $\mathrm{a}$ and use long or synthetic division. But can we find a way to reduce the possibilities for a first?

(9) If linear transformations can be represented by matrices, is there a matrix operation that corresponds to composition of linear transformations?
Questions such as these are designed to elicit heuristic thinking from students. As with heuristics themselves, they do not guarantee success. But they are characteristic of the way in which mathematicians think, and students of mathematics need to get in touch with human reasoning in mathematics as well as the mathematics itself.

\section{Classroom Suggestions}

Formulated as questions, two principal educational issues have been raised in this paper, namely: (1) How do we dispel myths that tend to block mathematical learning? (2) How do we get our students to incorporate heuristics into their own thinking? These are tough problems because there are no unique solutions, and optimal solutions are yet to be found, if they exist at all. What works best for one professor may not work well at all for another. Based on my own experience over a number of years in the mathematics classroom, here are some sug. gestions I would make:

(1) Encourage students to learn to carry on a conversation with themselves, using sequences of internalized leading "self-questions" that elicit heuristic thinking. To help accomplish this, ask lots of heuristic-oriented questions in class, making students active participants rather than passive spectators. Have them step back and examine their own thinking, comparing it with the thinking of good problem solvers, and then use heuristic-oriented questions and answers in their communication with others.

(2) Hold problem solution critiques occasionally at the blackboard, where a student presents his/her solution to the group (including comments on reasoning), and the group discusses the solution and the reasoning. This is like a painting critique in an art class or analysis of a musical composition, except here the masterpiece is a problem solution.

(3) Have students work in teams on a project (e.g. mathematical modeling, mini-research project, computer-supported solution). This helps them to actively recruit and observe the ideas of others, using their expertise when necessary. By sharing ideas, students practice communication. 
(4) Require some technical writing, preferably early in the student's mathematical careers. Often students have difficulty with mathematics because they have not taken the time to formulate precise questions. Have them write down questions or write out a complete problem solution or proof. This forces them to organize their thinking and it also improves their ability to communicate.

(5) Assign fewer exercises and more problems. Higher expectations of students, kept reasonable, usually lead to better performance.

Mathematical instruction needs to change. As mathematics professors, we need to dispel myths that block mathematical learning; we also need to get familiar with and communicate explicitly those heuristic processes that differentiate experts from novices in our field, so that our students will incorporate these thinking strategies into their own perception of what mathematics is really about. Through mutual discovery and invention, students and teachers together can trace the path of mathematical thought as a truly human endeavor. 